\title{
LOCAL AUTOMORPHISMS ARE DIFFERENTIAL OPERATORS ON SOME BANACH SPACES
}

\author{
JOHN C. WELlS AND CHARLES R. DePRIMA ${ }^{1}$
}

ABstract. If $E$ belongs to a certain category of Banach spaces (the $B^{\infty}$-smooth spaces) which include Hilbert spaces and if $F$ is any normed space, it is proved that any local linear automorphism of $C^{\infty}(E, F)$ is a differential operator. This generalizes a result of $J$. Peetre when $E=R^{n}$ and $F=R$.

1. A result of J. Peetre ([2], [3]) is the following characterization of linear partial differential operators:

A linear map $T$ of $C^{\infty}\left(R^{n}, R\right)$ into $C^{\infty}\left(R^{n}, R\right)$ is a linear "partial differential operator" if and only if $T$ is local i.e. for each $f \in C^{\infty}\left(R^{n}, R\right)$, $\operatorname{support}(T f) \subset \operatorname{support}(f)$.

It should be noted that by a linear partial differential operator $T$ is meant a collection $\left\{A_{\alpha}\right\} \subset C^{\infty}\left(R^{n}, R\right)$ such that the sets

$$
G_{\alpha}=\left\{x \in R^{n} \mid A_{\alpha}(x) \neq 0\right\}
$$

from a locally finite collection and such that $T(f)(x)=\sum_{\alpha} A_{\alpha}(x) D^{\alpha}(f)(x)$ for each $x \in R^{n}$ and each $f \in C^{\infty}\left(R^{n}, R\right)$. Here $\alpha=\left(\alpha_{1}, \ldots, \alpha_{n}\right)$ is a multi-index, $|\alpha|=\sum_{i=1}^{n} \alpha_{i}$ and

$$
D^{\alpha}=\partial^{|\alpha|} / \partial x_{1}^{\alpha_{1}} \cdots \partial x_{n}^{\alpha_{n}}
$$

In this paper we prove that at least for $E$ in a certain category of Banach spaces this theorem extends to local (linear) automorphisms on $C^{\infty}(E, F)$ where $C^{\infty}(E, F)$ now denotes the infinitely Fréchet differentiable $F$-valued functions on $E$ and $F$ is any normed linear space. Defining $L_{s}^{k}(E, F)$ to be the bounded symmetric $k$-multilinear maps from $E$ to $F$ we have $D^{k} f(x) \in L_{s}^{k}(E, F)$ for each $x$.

A natural generalization of a finite dimensional differential operator to

Presented to the Society, January 25, 1973; received by the editors December 6, 1972 and, in revised form, January 12, 1973.

AMS (MOS) subject classifications (1970). Primary 47F05; Secondary 58G99, $58 \mathrm{~B} 10$.

Key words and phrases. Partial differential operators, local linear maps, smooth Banach spaces.

${ }^{1}$ Supported in part by NSF Grant GP-23392.

e. American Mathematical Society 1973 
an arbitrary Banach space is

$$
T(f)(x)=\sum_{i=0}^{\infty} \alpha_{i}(x)\left(D^{i} f(x)\right)
$$

where $\alpha_{i} \in C^{\infty}\left(E, L\left(L_{s}^{k}(E, F), F\right)\right)$ and the supports of the $\alpha_{i}$ form a locally finite collection. Such maps are clearly local linear automorphisms on $C^{\infty}(E, F)$.

As in Wells [6] we let

$$
B^{k}(E, F)=\left\{f \mid f \in C^{k}(E, F), \sup _{x \neq y}\left\|D^{k} f(x)-D^{k} f(y)\right\| /\|x-y\|<\infty\right\}
$$

and

$$
B^{\infty}(E, F)=\bigcap_{k=0}^{\infty} B^{k}(E, F)
$$

An isomorphic invariant of a Banach space due to Bonic and Frampton [1] is $C^{p}$ smoothness. $E$ is $C^{p}$ smooth, $p=0,1,2, \cdots, \infty$, if there is some $\eta \in C^{p}(E, R)$ with $\eta(0) \neq 0$ and $\eta(\{x \mid\|x\| \geqq 1\})=0$. Similarly, as in [6], $E$ will be called $B^{p}$ smooth, $j=0,1,2, \ldots, \infty$, if there is an $\eta \in B^{p}(E, R)$ with $\eta(0) \neq 0$ and $\eta(\{x \mid\|x\| \geqq 1\})=0 . B^{\infty}$ smooth spaces have been called uniformly $C^{\infty}$ smooth in Quinn. [4]. Finite dimensional spaces as well as $\mathscr{L}^{p}$ for $p$ an even integer are $B^{\infty}$ smooth. $l^{1}$ is not $C^{1}$ smooth. $c_{0}$ is $C^{\infty}$ smooth but not $B^{1}$ smooth. Separable $C^{p}$ or $B^{p}$ smooth Banach spaces admit partitions of unity of class $C^{p}$ or $B^{p}$ respectively. In these cases $C^{p}(E, F)$ or $B^{p}(E, F)$ is dense in $C^{0}(E, F)$ or $B^{0}(E, F)$ respectively for any $B$-space $F$. Refer to Bonic and Frampton [1], Wells ([5] and [6]) for more details.

2. THEOREM. If $E$ and $F$ are Banach space, if $E$ is $B^{\infty}$ smooth and if $T: C^{\infty}(E, F) \rightarrow C^{\infty}(E, F)$ is a local linear map, then $T$ is a differential operator in the sense described above.

The proof will require three lemmas. Only the first will use the $B^{\infty}$ smoothness of $E$. We will use $K_{r}(x)$ to denote the open ball of radius $r$ centered at $x$.

LEMMA 1. Let $x_{0} \in E$. There is a neighborhood $U_{x_{0}}$ of $x_{0}$ and an integer $k$ with that property that if $f, g \in C^{\infty}(E, F), y \in U_{x_{0}}$ and $D^{i} f(y)=D^{i} g(y)$ for $0 \leqq i \leqq k$ then $T(f)(y)=T(g)(y)$.

Proof. If this were not the case there would be an $x_{0} \in E$, a sequence $x_{n}$ tending to $x_{0}$ and a sequence $f_{n} \in C^{\infty}(E, F)$ with $D^{k} f_{n}\left(x_{n}\right)=0$ for $k \leqq n$ and $\left\|T\left(f_{n}\right)\left(x_{n}\right)\right\|=n$. By the $B^{\infty}$ smoothness there exists an $\eta \in B^{\infty}(E, R)$ with $\eta\left(\operatorname{cl}\left(K_{1 / 2}(0)\right)\right)=1$ and $\eta(\{x \mid\|x\| \geqq 1\})=0$. Let $N_{i}=\sup _{x}\left\|D^{i} \eta(x)\right\|$. For 
each $n$ there is an $M_{n}$ and an $r_{n}$ such that $\left\|D^{j} f_{n}(x)\right\| \leqq M_{n}\left(\left\|x-x_{n}\right\|\right)^{n+1-j}$ for $x \in K_{r_{n}}\left(x_{n}\right)$ and $0 \leqq j \leqq n$. Whenever $1 / a_{n}<r_{n}$ we have

$\sup _{x}\left\|D^{j}\left(f_{n}(x) \eta\left(a_{n}\left(x-x_{n}\right)\right)\right)\right\|$

$$
\begin{aligned}
& \leqq \sum_{i=0}^{j}\left(\begin{array}{l}
j \\
i
\end{array}\right) \sup _{x \in K_{1} / a_{n}\left(x_{n}\right)}\left\|D^{j-i} f_{n}(x)\right\| \cdot a_{n}^{i} \cdot \sup _{x}\left\|D^{i}(\eta(x))\right\| \\
& \leqq \sum_{i=0}^{j}\left(\begin{array}{l}
j \\
i
\end{array}\right) M_{n} \cdot\left(\frac{1}{a_{n}}\right)^{n+1-j+i} \cdot a_{n}^{i} \cdot N_{i}=\left(\frac{1}{a_{n}}\right)^{n+1-j} M_{n} \sum_{i=0}^{j}\left(\begin{array}{l}
j \\
i
\end{array}\right) N_{i} .
\end{aligned}
$$

Thus we can choose a sequence $a_{n}$ so that

(i) $1 / a_{n}<r_{n}$,

(ii) $K_{1 / a_{n}}\left(x_{n}\right) \cap K_{1 / a_{m}}\left(x_{m}\right)=\varnothing$ for $n \neq m$,

(iii) $\sup _{j \leqq n, x \in E}\left\|D^{j}\left(f_{n}(x) \eta\left(a_{n}\left(x-x_{n}\right)\right)\right)\right\|<\operatorname{dist}\left(x_{0}, K_{1 / a_{n}}\left(x_{n}\right)\right)$.

It follows that the function $f(x)=\sum_{n=1}^{\infty} f_{n}(x) \eta\left(a_{n}\left(x-x_{n}\right)\right)$ belongs to $C^{\infty}(E, F)$ and that $f(x) \equiv f_{n}(x)$ for $x \in K_{1 / 2 a_{n}}\left(x_{n}\right)$. Consequently

$$
\left\|T(f)\left(x_{n}\right)\right\|=n
$$

so that $T(f)$ is not a continuous function at $x_{0}$. This is a contradiction.

Let $E_{k}=F \oplus L_{s}^{1}(E, F) \oplus \cdots \oplus L_{s}^{k}(E, F)$. By Lemma 1 for each $x \in U_{x_{0}}$ there is a linear map $T_{x}: E_{k} \rightarrow F$ such that

$$
T(f)(x)=T_{x}\left(f(x), D f(x), \cdots, D^{k} f(x)\right) .
$$

In Lemmas 2 and 3, $x_{0}$ and $U_{x_{0}}$ will be fixed.

Lemma 2. $T_{x}$ is bounded except possibly at a set $I_{x_{0}}$ of isolated points of $U_{x_{0}}$.

Proof. If this were not the case there would exist a sequence $\left\{x_{n}\right\}$ with $\left\{x_{n}\right\} \subset U_{x_{0}}$ and a $y \in U_{x_{0}}$ with $y=\lim x_{n}$ and with $T_{x_{n}}$ unbounded for each $n$. Next we choose a collection $\left\{\varphi_{n}\right\} \subset B^{\infty}(E, R)$ with support $\varphi_{n} \cap$ support $\varphi_{m}=\varnothing$ for $n \neq m$, dist $\left(x_{0}\right.$, support $\left.\varphi_{n}\right)>0$ for all $n$, and $\varphi_{n}(x) \equiv$ 1 near $x_{n}$. (We observe that the $B^{\infty}$ smoothness of $E$ is not needed to construct the $\left\{\varphi_{n}\right\}$ since the $x_{n}$ 's can be separated by a disjoint collection of weak neighborhoods each of which is the support of a $B^{\infty}$ function equal to 1 near $x_{n}$.) For each $n$ choose $g_{n} \in C^{\infty}(E, F)$ such that

$$
\sup _{j \leqq n, x \in K_{1}\left(x_{0}\right)}\left\|D^{j}\left(g_{n}(x) \varphi_{n}(x)\right)\right\|<\operatorname{dist}\left(x_{0}, \text { support } \varphi_{n}\right)
$$

and $\left\|T_{x_{n}}\left(\left\{g_{n}\left(x_{n}\right), \cdots, D^{k} g_{n}\left(x_{n}\right)\right\}\right)\right\| \geqq n$. The function

$$
f(x)=\sum_{n=0}^{\infty} g_{n}(x) \varphi_{n}(x)
$$


belongs to $C^{\infty}(E, F)$ and $f(x)=g_{n}(x)$ near $x_{n}$. Consequently $\left\|T(f)\left(x_{n}\right)\right\| \geqq n$ which is impossible in view of the continuity of the function $T(f)$.

Thus $T_{x}$ induces a map $T^{0}: U_{x_{0}} \mid I_{x_{0}} \rightarrow L\left(E_{k}, F\right)$ such that $T^{0}(x)=T_{x}$. Hence $T^{0}(x)\left(f(x), \cdots, D^{k} f(x)\right)=T(f)(x)$ for $x \in U_{x_{0}} \backslash I_{x_{0}}$.

LEMMA 3. For each $p=0,1,2, \cdots$ and each $y_{0} \in U_{x_{0}}$ there is a neighborhood $U_{y_{0}}$ of $y_{0}$ such that $T^{0}\left|U_{y_{0}}\right| I_{x_{0}} \in B^{p}\left(U_{y_{0}} \mid I_{x_{0}}, L\left(E_{k}, F\right)\right)$.

PROOF. In Wells [6] it is shown that

$$
B^{p}(E, F)=\left\{f \mid f \in C^{0}(E, F), \sup _{x, h \neq 0}\left\|\Delta_{h}^{p+1} f(x)\right\| /\|h\|^{p+1}<\infty\right\}
$$

where $\Delta_{h} f(x)=f(x+h)-f(x)$. Suppose the lemma were false. Then for some $p$ and some $y_{0} \in U_{x_{0}}$ and for every neighborhood $N$ of $y_{0}$ contained in $x_{0}$, the supremum of $\left\|\Delta_{h}^{p+1} f(x)\right\| /\|h\|^{p+1}$ over all $x, h \neq 0$ with $x$, $x+h, \cdots, x+(p+1) h$ contained in $N \backslash I_{x_{0}}$ would be infinite. This would imply the existence of sequences $\left\{x_{n}\right\},\left\{h_{n}\right\}$ with $x_{n} \rightarrow y_{0}, h_{n} \rightarrow 0$,

and

$$
\left\{x_{n}, x_{n}+h_{n}, \cdots, x_{n}+(p+1) h_{n}\right\} \in U_{x_{0}} \backslash I_{x_{0}}
$$

$$
\left\|\Delta_{h_{n}}^{p+1} T^{0}\left(x_{n}\right)\right\| /\left\|h_{n}\right\|^{p+1} \geqq 4^{n} .
$$

Choose $A_{n} \in E_{k}$ with $\left\|A_{n}\right\|_{E_{k}} \leqq 3^{-n}$ and

$$
\left\|\Delta_{h n}^{p+1} T^{0}\left(x_{n}\right)\left(A_{n}\right)\right\| \geqq \frac{3}{4}\left\|\Delta_{h n}^{p+1} T^{0}\left(x_{n}\right)\right\| \cdot 3^{-n} .
$$

Since for any $t, s$ in a normed linear space $\sup \{\|t+\sigma s\| \mid \sigma= \pm 1\} \geqq\|s\|$, we may inductively choose $\sigma_{n}= \pm 1, n=1,2,3, \cdots$, so that

$$
\left\|\Delta_{h_{n}}^{p+1} T^{0}\left(x_{n}\right)\left(\sum_{j=1}^{n} \sigma_{j} A_{j}\right)\right\|>\frac{3}{4}\left\|\Delta_{h_{n}}^{p+1} T^{0}\left(x_{n}\right)\right\| \cdot 3^{-n} .
$$

For each $n$ let $g_{n}$ be the $k$ polynomial such that $A_{n}=\left\{g_{n}\left(x_{n}\right), \cdots, D^{k} g_{n}\left(x_{n}\right)\right\}$ and $f(x)$ be the $k$ polynomial $\sum_{i=1}^{\infty} \sigma_{j} g_{j}(x)$. Then

$$
\begin{aligned}
\left\|\Delta_{h_{n}}^{p+1} T(f)\left(x_{n}\right)\right\| & =\left\|\Delta_{h_{n}}^{p+1} T^{0}\left(x_{n}\right)\left(f\left(x_{n}\right), \cdots, D^{k} f\left(x_{n}\right)\right)\right\| \\
& \geqq\left\|\Delta_{h_{n}}^{p+1} T^{0}\left(x_{n}\right)\left(\sum_{j=1}^{n} \sigma_{j} A_{j}\right)\right\|-\left\|\Delta_{h_{n}}^{p+1} T^{0}\left(x_{n}\right)\left(\sum_{n+1}^{\infty} \sigma_{j} A_{j}\right)\right\| \\
& \geqq\left\|\Delta_{h_{n}}^{p+1} T^{0}\left(x_{n}\right)\right\| \cdot\left(\frac{3}{4} 3^{-n}-\sum_{j=n+1}^{\infty} 3^{-j}\right) \\
& =\frac{1}{4} 3^{-n} \cdot\left\|\Delta_{h_{n}}^{p+1} T^{0}\left(x_{n}\right)\right\| \geqq \frac{1}{4}(4 / 3)^{n} \cdot\left\|h_{n}\right\|^{p+1} .
\end{aligned}
$$

But this is a contradiction since, for every $p, T(f)$ is $B^{p}$ in some neighborhood of $y_{0}$. Q.E.D. 
We are now in a position to prove the theorem. First observe that the choice of $p=0$ in Lemma 3 implies that the exceptional set $I_{x_{0}}$ of Lemma 2 is void. Hence $T^{0}$ is defined on all of $U_{x_{0}}$ and by Lemma 3 is locally $B^{p}$ for any $p$ so that $T^{0} \in C^{\infty}\left(U_{x_{0}}, L\left(E_{k}, F\right)\right)$. Consequently there exist $\alpha_{n}^{0} \in$ $C^{\infty}\left(U_{x_{0}}, L\left(L_{s}^{n}(E, F), F\right)\right), n=0,1, \cdots, k$, such that

$$
T(f)(x)=\sum_{n=0}^{k} \alpha_{n}^{0}\left(D^{n} f(x)\right)
$$

for all $x \in U_{x_{0}}$. Suppose that $T(f)(x)=\sum_{n=0}^{k^{\prime}} \alpha_{n}^{\prime}\left(D^{n} f(x)\right)$ for $x \in U_{x_{1}}$ with $\alpha_{n}^{\prime} \in C^{\infty}\left(U_{x_{1}}, L\left(L_{s}^{n}(E, F), F\right)\right)$. Without loss of generality we may assume $k=k^{\prime}$. If $x \in U_{x_{0}} \cap U_{x_{1}}$ and $A \in L_{s}^{n}(E, F)$ for $n \leqq k$, then for $g(x)=(1 / n !)$ $A(x, x, \cdots, x)$ we find $\alpha_{n}^{0}(A)=T(g)(x)=\alpha_{n}^{\prime}(A)$. Hence on $U_{x_{0}} \cap U_{x_{1}}$, $\alpha_{n}^{0}$ and $\alpha_{n}^{\prime}$ agree, so that we may define maps

$$
\alpha_{n} \in C^{\infty}\left(E, L\left(L_{s}^{n}(E, F), F\right)\right), \quad n=0,1, \cdots,
$$

such that $(T f)(x)=\sum_{n=0}^{\infty} \alpha_{n}(x)\left(D^{n} f(x)\right)$ for $x \in E$ and the $\left\{\alpha_{n}\right\}$ have locally finite supports. Consequently $T$ is a differential operator.

\section{BIBLIOGRAPHY}

1. R. Bonic and J. Frampton, Smooth functions on Banach manifolds, J. Math. Mech. 15 (1966), 877-898. MR 33 \#6647.

2. Jaak Peetre, Une caractérisation abstraite des opérateurs différentiels, Math. Scand. 7 (1959), 211-218. MR 22 \#3001.

3. - Réctification à l'article une caractérisation abstraite des opérateurs différentiels, Math. Scand. 8 (1960), 116-120. MR 23 \#A1923.

4. F. Quinn, Transversal approximation on Banach manifolds, Proc. Sympos. Pure Math., vol. 15, Amer. Math. Soc., Providence, R.I., 1970. MR 41 \#9304.

5. J. Wells, Differentiable functions on $c_{0}$, Bull. Amer. Math. Soc. 75 (1969), 117-118. MR 38 \#2590.

6. - Differentiable functions on Banach spaces with Lipschitz derivatives, J. Differential Geometry (to appear).

Department of Mathematics, California State University, Northridge, CALIFORNIA 91324

Department of Mathematics, California Institute of Technology, Pasadena, California 91109 (Current address of C. R. DePrima)

Current address (John C. Wells): Mathematical Sciences Division, National Science Foundation, Washington, D.C. 20550 